\section{ECCOMAS}

Proceedia
EUROGEN 2021

$14^{\text {th }}$ ECCOMAS Thematic Conference on Evolutionary and Deterministic Methods for Design, Optimization and Control N. Gauger, K. Giannakoglou, M. Papadrakakis, J. Periaux (eds.) Streamed from Athens, Greece, 28-30 June 2021

\title{
AERODYNAMIC SHAPE OPTIMIZATION OF THE MEXICO WIND TURBINE BLADE USING THE CONTINUOUS ADJOINT METHOD
}

\author{
E. M. Papoutsis-Kiachagias ${ }^{1}$, M. Erfan Farhikhteh ${ }^{1}$, T. Skamagkis ${ }^{1}$, and \\ K. C. Giannakoglou ${ }^{1}$ \\ ${ }^{1}$ National Technical University of Athens, \\ Parallel CFD \& Optimization Unit, School of Mechanical Engineering \\ Zografou Campus, 9 Iroon Polytechniou Str \\ e-mail: \{vpapout,mohammaderfanfarhikhteh,themistoklissk, kgianna\}@ mail.ntua.gr
}

\begin{abstract}
This paper presents the aerodynamic shape optimization of the MEXICO wind turbine (WT) blade, targeting the maximization of the axial moment and, hence, of the produced power. The optimization is conducted using the OpenFOAM-based continuous adjoint solver named adjointOptimisation Foam, developed and made publicly available by the group of authors. This implements and solves the adjoint to the Navier-Stokes system of equations, coupled with the differentiation of the Spalart-Allmaras turbulence model. Herein, this was extended to include the adjoint to the flow equations which are solved for the absolute velocity in the relative reference frame. Challenges in the convergence of the adjoint equations, mostly attributed to flow unsteadiness causing marginal convergence of the steady flow solver, are treated by additionally implementing the Recursive Projection Method (RPM). Assessment of the adjoint sensitivities with finite differences in a similar 2D case is also included. Then, the flow solution for the MEXICO WT case is compared with the outcome of another CFD solvers and experimental data, prior to the application of the expanded optimization software to maximize the axial moment of the WT. The blade and the displacement of the surrounding grid nodes are parameterized using a volumetric B-Splines morphing box. The optimization designed a blade bended in the axial direction axial moment, having a higher by $10.8 \%$.
\end{abstract}

Keywords: Aerodynamic Optimization, Continuous Adjoint, MEXICO Wind Turbine, Recursive Projection Method. 


\section{INTRODUCTION}

Wind energy is a widely used renewable energy source producing green and clean energy [1]. Among other, an increased interest has surfaced on how to decrease the cost of the produced energy by wind turbines (WTs). This can be achieved by reducing the turbine capital cost and/or by increasing the annual energy production. Upscaling WTs to have a bigger swept area is a way to extract more energy, however, this solution affects the structural mechanics of the WT [2], since mass increases with the cube of the rotor radius. Generally, the design of WTs is a multidisciplinary trade-off among aerodynamic performance, structural efficiency and manufacturing cost [3].

Currently, low-fidelity models are often used to design WT blades, because of their low computational cost and simple implementation. Among them, Blade Element Momentum (BEM) models are the most widely used [4]. Depending on the application however, such a model may have limited success in accurately capturing viscous flow effects, compressibility and complex 3D patterns; hence, 3D Computational Fluid Dynamics (CFD) software should be used instead.

In other areas, such as aerospace or the automotive industry, CFD-based aerodynamic shape optimization is used on a regular basis $[5,6]$. Similar methods in the wind energy field are not yet in widespread use. High-fidelity CFD-based shape optimization can be performed with or without computing the gradient of the objective function. In [7], a WT blade winglet was optimized using a 3D CFD model and a gradient-free method using two design variables to increase the moment which, in turn, increased power production by $9 \%$. Increasing the number of design variables is expected to increase the optimization turn-around time of gradient-free methods a lot. Gradient-based optimization may overcome this manner as the adjoint method is the only way to compute the required gradient components at a cost that does not scale with their number $[8,9]$. The first time the adjoint to a RANS equations solver was used to optimize the lift-to-drag ratio of a WT blade airfoil was in [10].

A few 3D adjoint-based shape optimizations considered modelling the rotation effects. In one of them [11], the NREL Phase VI rotor was optimized using the RANS equations. The continuous adjoint to the discrete adjoint to the RANS equations, including the adjoint to the Spalart-Allmaras turbulence model, was used in [12] to maximize the power of the MEXICO WT rotor with the flow and adjoint solvers running on GPUs.

In the present work, the aerodynamic shape of the MEXICO WT blade is optimized in order to increase the axial moment and, thus, power production. The RANS equations are formulated in the relative reference frame and solved for the absolute velocity. Initially, the CFD analysis of the MEXICO WT blade is validated with two other CFD solvers and experimental data. A continuous adjoint formulation is then used to compute the gradient and support the optimization. The adjoint to the terms emerging from changing the reference frame to the relative one is also included, along with the differentiation of the Spalart-Allmaras turbulence model. Although a steady-state RANS solver was used and was sufficient in capturing the main aspects of the flow, small scale unsteadiness led to oscillating flow solver residuals and, as a result, the adjoint solver diverged on most optimization cycles. To stabilize the latter and allow an uninterrupted optimization, the Recursive Projection Method (RPM), developed in [13] for the stabilization of unstable iterative procedures, was used to support the adjoint method, leading to an increased axial moment by $10.8 \%$. 


\section{Flow and adjoint equations}

\subsection{Flow (primal) equations}

The flow model includes the steady-state RANS equations for incompressible flows, coupled with the Spalart-Allmaras turbulence model [14]. The flow equations are solved for the absolute velocity in the rotating reference frame. These equations coincide with those of the Multiple Reference Frame (MRF) approach, with uniform angular speed $₫$ being uniform along the entire computational domain in our case. The flow equations read

$$
\begin{aligned}
& R^{p}=-\frac{\partial w_{j}}{\partial x_{j}}=0 \\
& R_{i}^{v}=w_{j} \frac{\partial v_{i}}{\partial x_{j}}-\frac{\partial \tau_{i j}}{\partial x_{j}}+\frac{\partial p}{\partial x_{i}}+e_{i j k} \varpi_{j} v_{k}=0, \quad i=1,2,3 \\
& R^{\widetilde{\nu}}=w_{j} \frac{\partial \widetilde{\nu}}{\partial x_{j}}-\frac{\partial}{\partial x_{j}}\left[\left(\nu+\frac{\widetilde{\nu}}{\sigma}\right) \frac{\partial \widetilde{\nu}}{\partial x_{j}}\right]-\frac{c_{b 2}}{\sigma}\left(\frac{\partial \widetilde{\nu}}{\partial x_{j}}\right)^{2}-\widetilde{\nu} P(\widetilde{\nu})+\widetilde{\nu} D(\widetilde{\nu})=0 \\
& R^{\Delta}=\frac{\partial}{\partial x_{j}}\left(\frac{\partial \Delta}{\partial x_{j}} \Delta\right)-\Delta \frac{\partial^{2} \Delta}{\partial x_{j}^{2}}-1=0
\end{aligned}
$$

where $v_{i}$ and $w_{i}$ are the absolute and relative velocity components, respectively $\left(v_{i}=w_{i}+\right.$ $e_{i j k} \varpi_{j}\left(x_{k}-x_{k}^{c}\right)$ where $x_{k}$ denotes the position vector, $e_{i j k}$ stands for the Levi-Civita symbol and $x_{k}^{C}$ corresponds to the origin lying on the rotation axis), $p$ is the static pressure divided by the constant fluid density, $\tau_{i j}=\left(\nu+\nu_{t}\right)\left(\frac{\partial v_{i}}{\partial x_{j}}+\frac{\partial v_{j}}{\partial x_{i}}\right)$ and $\nu$ and $\nu_{t}=\widetilde{\nu} f_{v_{1}}$ are the constant bulk and turbulent viscosities. Eq. 1c is solved for $\widetilde{\nu}$ and terms $P(\widetilde{\nu}) \& D(\widetilde{\nu})$ stand for the production and destruction terms, respectively, while the rest of terms in Eq. 1c are explained in [14]. Eq. $1 \mathrm{~d}$ is the eikonal equation, [15], used to compute distances, $\Delta$, from the nearest wall, as required by the turbulence model. The objective function to be maximized is the axial moment coefficient,

$$
J=\frac{\int_{S_{W}} r_{i}^{M} e_{i j k}\left(x_{j}-x_{j}^{c}\right)\left(-\tau_{k l} n_{l}+p n_{k}\right) d S}{\frac{1}{2} l A U_{\infty}^{2}}
$$

where $r_{i}^{M}$ is the unit vector in the axial direction. The blade length is $l, U_{\infty}$ is the far-field velocity magnitude, $A$ is the blade area perpendicular to the flow direction and $S_{W}$ is the blade surface.

\subsection{Continuous adjoint formulation}

The first step in the formulation of the continuous adjoint method is the definition and subsequent differentiation of the Lagrangian function

$$
L=J+\int_{\Omega}\left(u_{i} R_{i}^{v}+q R^{p}+\widetilde{\nu_{a}} R^{\widetilde{\nu}}+\Delta_{a} R^{\Delta}\right) d \Omega
$$

In Eq. 3, $u_{i}$ denotes the adjoint velocity components, $q$ is the adjoint to the pressure $p, \widetilde{\nu_{a}}$ is the adjoint turbulence variable and $\Delta_{a}$ is the adjoint distance. The derivatives of $L$ with respect to (w.r.t.) the design variables $b_{n}, n \in[1, N]$, yields

$$
\frac{\delta L}{\delta b_{n}}=\frac{\delta J}{\delta b_{n}}+\int_{\Omega}\left(u_{i} \frac{\delta R_{i}^{v}}{\delta b_{n}}+q \frac{\delta R^{p}}{\delta b_{n}}+\widetilde{\nu_{a}} \frac{\delta R^{\widetilde{\nu}}}{\delta b_{n}}+\Delta_{a} \frac{\delta R^{\Delta}}{\delta b_{n}}\right) d \Omega
$$


where $\frac{\delta}{\delta b_{n}}($.$) represents total (or material) derivatives and$

$$
\begin{aligned}
\frac{\delta J}{\delta b_{n}} & =\frac{1}{\frac{1}{2} l A U_{\infty}^{2}}\left[\int_{S_{W}} r_{i}^{M} e_{i j k}\left(-\tau_{k l} n_{l}+p n_{k}\right) \frac{\delta x_{j}}{\delta b_{n}} d S-\int_{S_{W}} r_{i}^{M} e_{i j k}\left(x_{j}-x_{j}^{c}\right) \frac{\delta \tau_{k l}}{\delta b_{n}} n_{l} d S\right. \\
& \left.+\int_{S_{W}} r_{i}^{M} e_{i j k}\left(x_{j}-x_{j}^{c}\right)\left(-\tau_{k l}+p \delta_{k}^{l}\right) \frac{\delta\left(n_{l} d S\right)}{\delta b_{n}}+\int_{S_{W}} r_{i}^{M} e_{i j k}\left(x_{j}-x_{j}^{c}\right) \frac{\delta p}{\delta b_{n}} n_{k} d S\right]
\end{aligned}
$$

Further developing Eq. 4 makes use of $\frac{\delta}{\delta b_{n}}\left(\frac{\partial(.)}{\partial x_{j}}\right)=\frac{\partial}{\partial x_{j}}\left(\frac{\delta(.)}{\delta b_{n}}\right)-\frac{\partial(.)}{\partial x_{k}} \frac{\partial}{\partial x_{j}}\left(\frac{\delta x_{k}}{\delta b_{n}}\right)$ (see [16]), and the Gauss divergence theorem. Only the differentiation of the continuity equation is shown

$$
\begin{aligned}
\int_{\Omega} q \frac{\delta R^{p}}{\delta b_{n}} d \Omega & =\int_{S_{W}}-q n_{j} \frac{\delta w_{j}}{\delta b} d S \\
& +\int_{\Omega}\left[\frac{\partial q}{\partial x_{j}} \frac{\delta v_{j}}{\delta b_{n}}-\frac{\partial q}{\partial x_{j}} e_{j l k} \varpi_{l} \frac{\delta x_{k}}{\delta b_{n}}+q \frac{\partial v_{j}}{\partial x_{k}} \frac{\partial}{\partial x_{j}}\left(\frac{\delta x_{k}}{\delta b_{n}}\right)-q e_{j l k} \varpi_{l} \frac{\partial}{\partial x_{j}}\left(\frac{\delta x_{k}}{\delta b_{n}}\right)\right] d \Omega
\end{aligned}
$$

In Eq. 6, all terms including the so-called grid sensitivities $\frac{\delta x_{k}}{\delta b_{n}}$ contribute to the sensitivity derivatives. The rest of integrals in Eq. 3 are expanded similary. Boundary integrals including flow variations contribute to the adjoint boundary conditions whereas field integrals of the same quantities contribute to the field adjoint equations.

\subsubsection{Field adjoint equations}

In order to avoid the computation of variations in the flow variables w.r.t. $b_{n}$ within the field integrals of the developed form of Eq. 4, the multipliers of these terms are set to zero. Thus, the field adjoint equations

$$
\begin{aligned}
R^{q} & =-\frac{\partial u_{j}}{\partial x_{j}}=0 \\
R_{i}^{u} & =u_{j} \frac{\partial v_{j}}{\partial x_{i}}-\frac{\partial\left(w_{j} u_{i}\right)}{\partial x_{j}}-\frac{\partial \tau_{i j}^{a}}{\partial x_{j}}+\frac{\partial q}{\partial x_{i}}+e_{i j k} u_{j} \varpi_{k}+\widetilde{\nu_{a}} \frac{\partial \widetilde{\nu}}{\partial x_{i}} \\
& -\frac{\partial}{\partial x_{l}}\left(\widetilde{\nu_{a}} \widetilde{\mathcal{C}_{Y}} \frac{\partial v_{k}}{Y} e_{m j k} \frac{\nu_{m l i}}{\partial x_{j}}\right)=0, i=1,2,3 \\
R^{\widetilde{\nu_{a}}} & =-\frac{\partial\left(w_{j} \widetilde{\nu_{a}}\right)}{\partial x_{j}}-\frac{\partial}{\partial x_{j}}\left[\left(\nu+\frac{\widetilde{\nu}}{\sigma}\right) \frac{\partial \widetilde{\nu_{a}}}{\partial x_{j}}\right]+\frac{1}{\sigma} \frac{\partial \widetilde{\nu_{a}}}{\partial x_{j}} \frac{\partial \widetilde{\nu}}{\partial x_{j}} \\
& +2 \frac{c_{b 2}}{\sigma} \frac{\partial}{\partial x_{j}}\left(\widetilde{\nu_{a}} \frac{\partial \widetilde{\nu}}{\partial x_{j}}\right)+\widetilde{\nu_{a}} \widetilde{\mathcal{C}}+\frac{\partial \nu_{t}}{\partial \widetilde{\nu}} \frac{\partial u_{i}}{\partial x_{j}}\left(\frac{\partial v_{i}}{\partial x_{j}}+\frac{\partial v_{j}}{\partial x_{i}}\right)+(-P+D) \widetilde{\nu_{a}}=0 \\
R^{\Delta_{a}} & =-2 \frac{\partial}{\partial x_{j}}\left(\Delta_{a} \frac{\partial \Delta}{\partial x_{j}}\right)+\widetilde{\nu} \widetilde{\nu_{a}} \mathcal{C}_{\Delta}=0
\end{aligned}
$$


emerge, where $\tau_{i j}^{a}=\left(\nu+\nu_{t}\right)\left(\frac{\partial u_{i}}{\partial x_{j}}+\frac{\partial u_{j}}{\partial x_{i}}\right)$ is the adjoint stress tensor. The $C_{\widetilde{\nu_{a}}}, C_{Y}$ and $C_{\Delta}$ terms in Eq. 7c are defined in [17].

\subsection{Sensitivity derivatives}

After eliminating all other integrals in the developed form of Eq. 4, by satisfying the adjoint field equations and boundary conditions (omitted here in the interest of space; see [9] for a detailed derivation), the remaining terms can be computed at a negligible cost and constitute the sensitivity derivatives of $J$, which read

$$
\begin{aligned}
\frac{\delta L}{\delta b_{n}} & =\int_{S_{W}} \tau_{i j}^{a} n_{j} e_{i k l} \varpi_{k} \frac{\delta x_{l}}{\delta b_{n}} d S+\frac{1}{\frac{1}{2} l A U_{\infty}^{2}}\left[\int_{S_{W}} r_{i}^{M} e_{i j k}\left(-\tau_{k l} n_{l}+p n_{k}\right) \frac{\delta x_{j}}{\delta b_{n}} d S\right. \\
& \left.-\int_{S_{W}} r_{i}^{M} e_{i j k}\left(x_{j}-x_{j}^{c}\right)\left(\tau_{k l}+p \delta_{k}^{l}\right) \frac{\delta\left(n_{l} d S\right)}{\delta b_{n}}\right]-\int_{\Omega}\left(\frac{\partial q}{\partial x_{j}}+u_{i} \frac{\partial v_{i}}{\partial x_{j}}+\widetilde{\nu_{a}} \frac{\partial \widetilde{\nu}}{\partial x_{j}}\right) e_{j l k} \varpi_{l} \frac{\delta x_{k}}{\delta b_{n}} d \Omega \\
& -\int_{\Omega}\left[q \frac{\partial v_{j}}{\partial x_{k}}+q e_{j l k} \varpi_{l}+u_{i} w_{j} \frac{\partial v_{i}}{\partial x_{j}}+\tau_{i j}^{a} \frac{\partial v_{i}}{\partial x_{k}}+u_{j} \frac{\partial p}{\partial x_{k}}+\widetilde{\nu_{a}} w_{j} \frac{\partial \widetilde{\nu}}{\partial x_{k}}+\left(\nu+\frac{\widetilde{\nu}}{\sigma}\right) \frac{\partial \widetilde{\nu_{a}}}{\partial x_{j}} \frac{\partial \widetilde{\nu}}{\partial x_{k}}\right. \\
& \left.-2 \widetilde{\nu_{a}} \frac{c_{b 2}}{\sigma} \frac{\partial \widetilde{\nu}}{\partial x_{j}} \frac{\partial \widetilde{\nu}}{\partial x_{k}}+\widetilde{\nu_{a}} \widetilde{\nu} \mathcal{Y} \frac{Y_{q}}{\|\vec{Y}\|} e_{q j l} \frac{\partial v_{l}}{\partial x_{k}}+2 \Delta_{a} \frac{\partial \Delta}{\partial x_{j}} \frac{\partial \Delta}{\partial x_{k}}\right] \frac{\partial}{\partial x_{j}}\left(\frac{\delta x_{k}}{\delta b_{n}}\right) d \Omega
\end{aligned}
$$

where $\vec{Y}$ is the flow vorticity.

\section{The Recursive Projection Method}

Both the primal and adjoint equations are solved in OpenFOAM using a pressure-based solver. The primal and adjoint solvers are both variants of the standard SIMPLE algorithm [18] with small modifications. The slow convergence, or even divergence, of SIMPLE-like solvers is usually related to a difficulty in reducing the primal/adjoint pressure equation residuals. This difficulty is even more pronounced in cases that exhibit unsteadiness where it is not unusual for the steady primal solver residuals to marginally converge and then begin to oscillate after a number of iterations. This is usually accompanied by the subsequent divergence of the continuous adjoint solver; this behavior may be observed even in cases with small-scale unsteadiness [19]. This issue was observed in the optimisation of the WT and, on a number of optimization cycles, the use of the RPM was necessary to make the adjoint equations converge.

The RPM is a technique for stabilizing unstable iterative procedures, formally written as $\boldsymbol{U}^{(n+1)}=\boldsymbol{F}\left(\boldsymbol{U}^{(n)}\right)$, where $\boldsymbol{U} \in \mathbb{R}^{M}$ is the array of (primal or adjoint) unknowns and $n$ the iteration counter. If the largest eigenvalue of the Jacobian matrix $F_{U}$ exceeds unity, such a scheme is expected to diverge and, on many occasions, the RPM can make such an otherwise diverging scheme converge. First, the method needs to identify the diverging modes of $F_{U}$, i.e. the eigenvectors corresponding to the largest eigenvalues. Once identified, and assuming that these modes are $m$ in total, they can form a basis $Z \in \mathbb{R}^{M \times m}$ which is used to split the solution space $\mathbb{R}^{M}$ into two parts: the unstable subspace $\mathbb{P} \in \mathbb{R}^{M}$, spanned by the $m$ diverging modes of $F_{U}$, and its orthogonal complement $\mathbb{Q} \in \mathbb{R}^{M}$. The solution $U$ is also decomposed into $\mathrm{U}_{p} \in \mathbb{P}$ and $\mathbf{U}_{q} \in \mathbb{Q}$; the unstable and stable parts of the solution respectively. A stabilized iterative scheme is thus derived where a Newton iteration is performed on $\mathbf{U}_{p}$ while the original scheme is retained for $\mathbf{U}_{q}$. This way, the RPM makes a previously diverging scheme converge.

Practically, $m$ is initially zero and gradually grows as diverging and slowly decaying modes are identified and incrementally appended to $Z$ throughout the solution of the adjoint equations. 
Additionally, $Z$ is approximated using power iterations on an initial matrix estimate (more details about the implementation of the RPM can be found in [13]). Finally, the new scheme derived through the RPM becomes stable after all the diverging modes have been identified and provided that $Z$ has been approximated with sufficient accuracy.

\section{Analysis and Optimization of the WT blades}

This work presents the aerodynamic shape optimization of a Horizontal Axis Wind Turbine (HAWT) blade, namely that of the MEXICO WT, associated with the EU project "Model Rotor Experiments In Controlled Conditions" [20]. Measurements were performed in the Large LowSpeed Facility of DNW in the Netherlands $[20,21]$. The computational domain $\Omega$ includes one third of the WT disk, with periodic boundary conditions. The CFD domain and mesh are presented in Fig. 1. The hybrid CFD mesh is generated with $\approx 10^{7}$ cells using Pointwise.
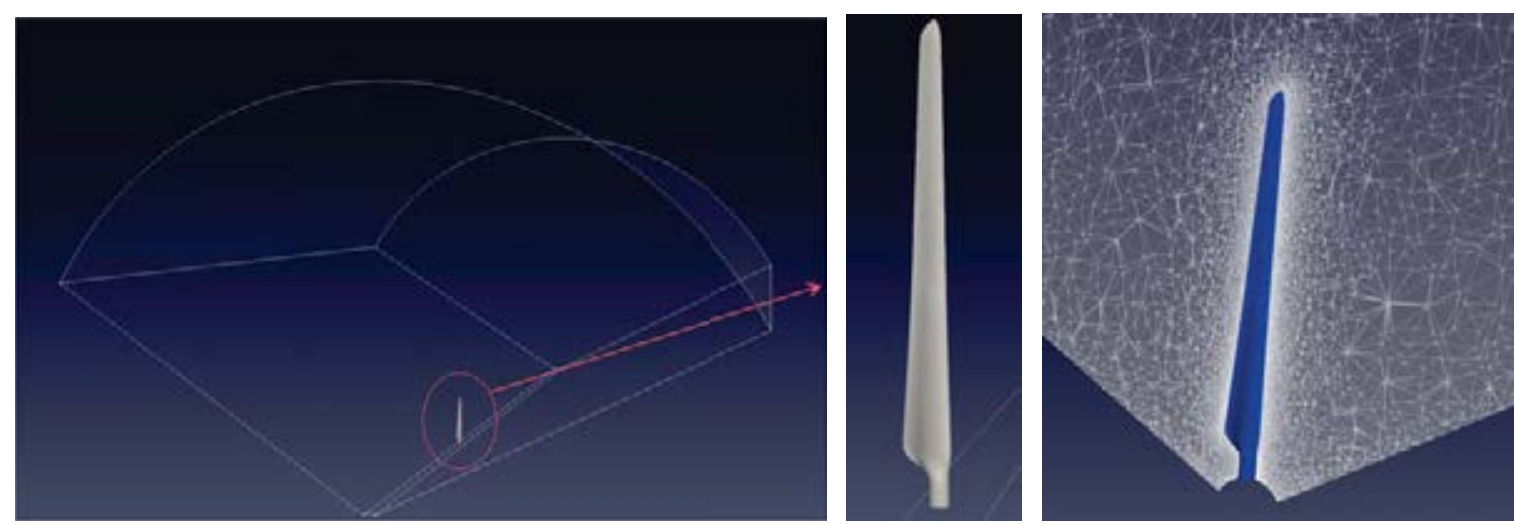

Figure 1: CFD domain and mesh around the MEXICO WT blade.

\subsection{Flow Solver Verification}

The wind speed and yaw angle are $10 \mathrm{~m} / \mathrm{s}$ and $0^{\circ}$, respectively. The pressure coefficient distribution on a number of different spanwise positions over the blade is shown in Fig. 2. OpenFOAM-based results are compared with wind tunnel measurements and two other CFD results. The latter are obtained from the MaPFlow code of the Lab. of Aerodynamics, NTUA [22] and the GPU-enabled PUMA code (incompressible flow solver) of the Parallel CFD \& Optimization Unit, NTUA [12]. All CFD results are in very good agreement. 


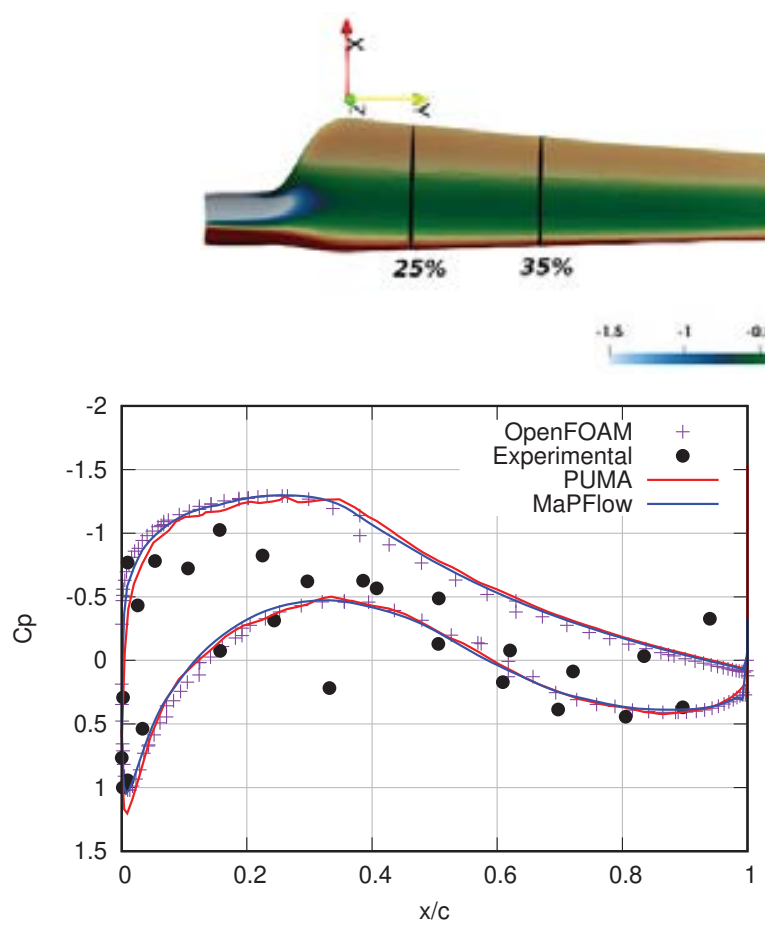

(a) $25 \% \mathrm{Span}$

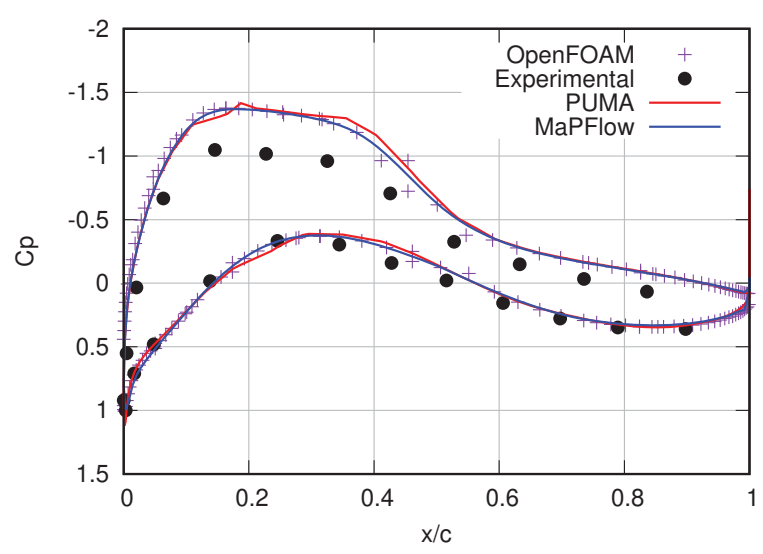

(c) $60 \%$ Span

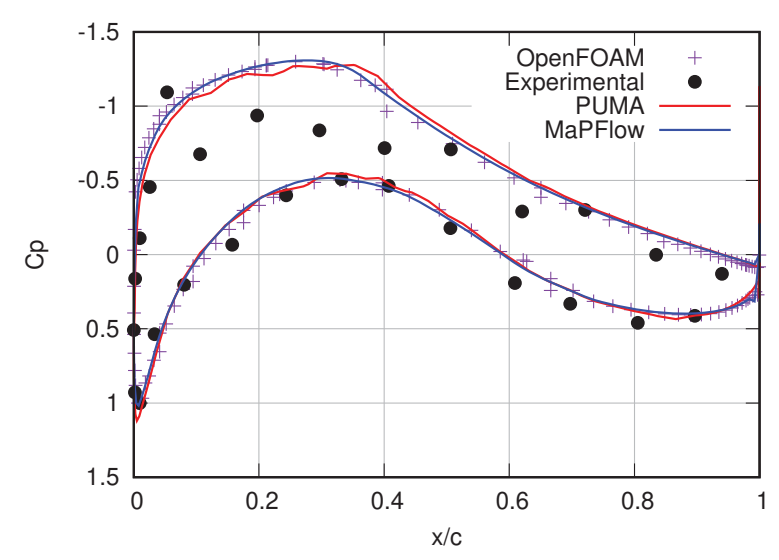

(b) $35 \%$ Span

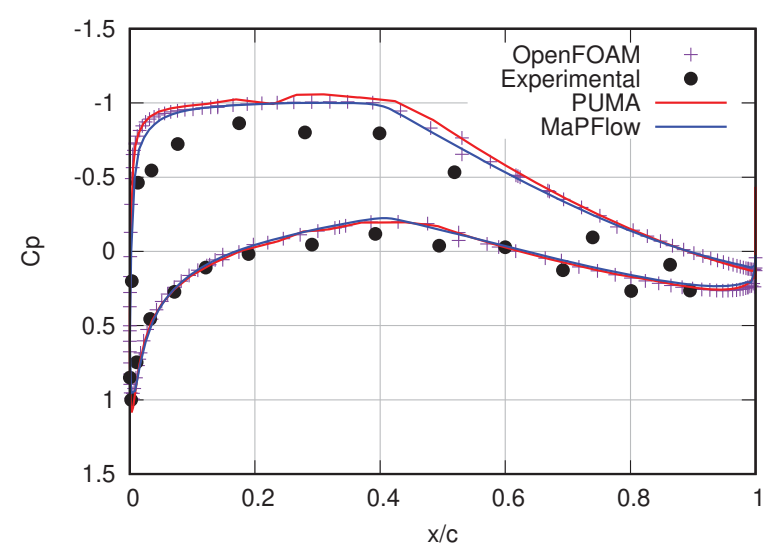

(d) $82 \%$ Span

Figure 2: MEXICO WT Blade: Comparison of the pressure coefficient computed by OpenFOAM, PUMA and MaPFlow with measurements, at four spanwise positions.

It can be observed that pressure on the suction side is underpredicted by all CFD results and, in those cases, the experimental results are not in good agreement with CFD. A number of possible reasons for this are mentioned in [23]. On the other hand, all CFD results shown here and additional ones presented in [23] are in very good agreement.

\subsection{Verification of the Sensitivity Derivatives}

The adjoint-based sensitivity derivatives are verified against the results of finite-differences (FDs) in this section. Since FDs are very time consuming due to the need to solve the flow 
equations twice for each design variable, the adjoint-based sensitivity derivatives are verified on a 2D mixer case, Fig. 3. Adjoint-based sensitivities are in a good agreement with FDs and can be used in a gradient-based optimization loop.

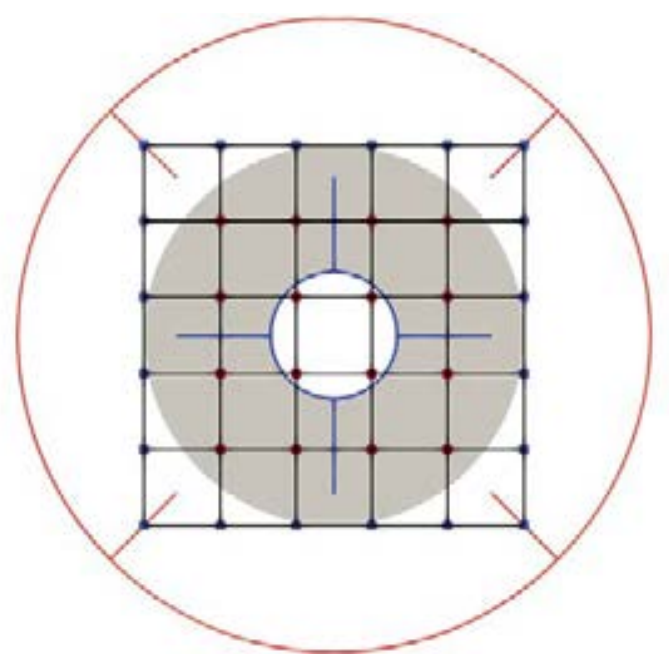

(a)

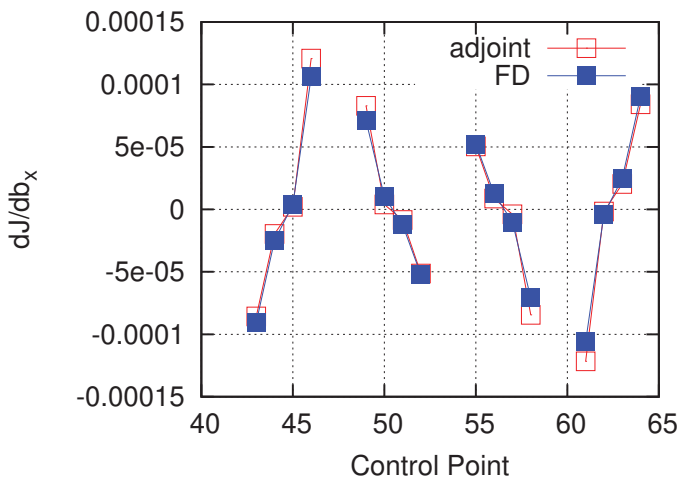

(b)

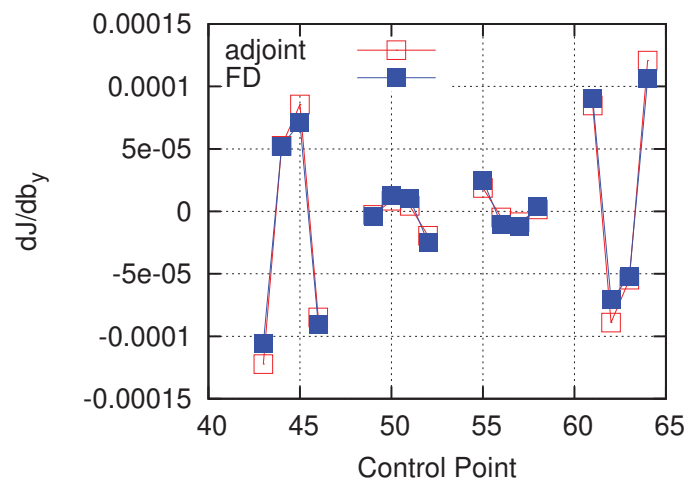

(c)

Figure 3: 2D mixer: Verification of the adjoint-based sensitivity derivatives with FDs. (a) Case geometry; blue/red lines correspond to rotating/stationary walls. The grey area indicates the rotating part of the computational domain, i.e. the part in which additional terms in the momentum equations, Eq. $1 \mathrm{~b}$ are introduced. The lattice of control points parameterizing the rotor is also shown. The coordinates of the control points in red act as the design variables. (b)-(c) Comparison of the adjoint-based derivatives with FDs for the $x$ and $y$ coordinates of the control points.

\subsection{Wind Turbine Blade Optimization}

After validating the flow solver and sensitivity derivatives, the next step includes the optimization of the WT blade to maximize the axial moment coefficient, Eq. 2. The blade was parameterized using the volumetric B-Splines morphing box presented in Fig. 4. The role undertaken by the RPM within the first optimization cycle is explained in Fig. 5. With a converged adjoint, optimization ensued and, after 15 cycles, the axial moment coefficient has increased by $10.8 \%$, from 0.2332 to 0.2584 . 


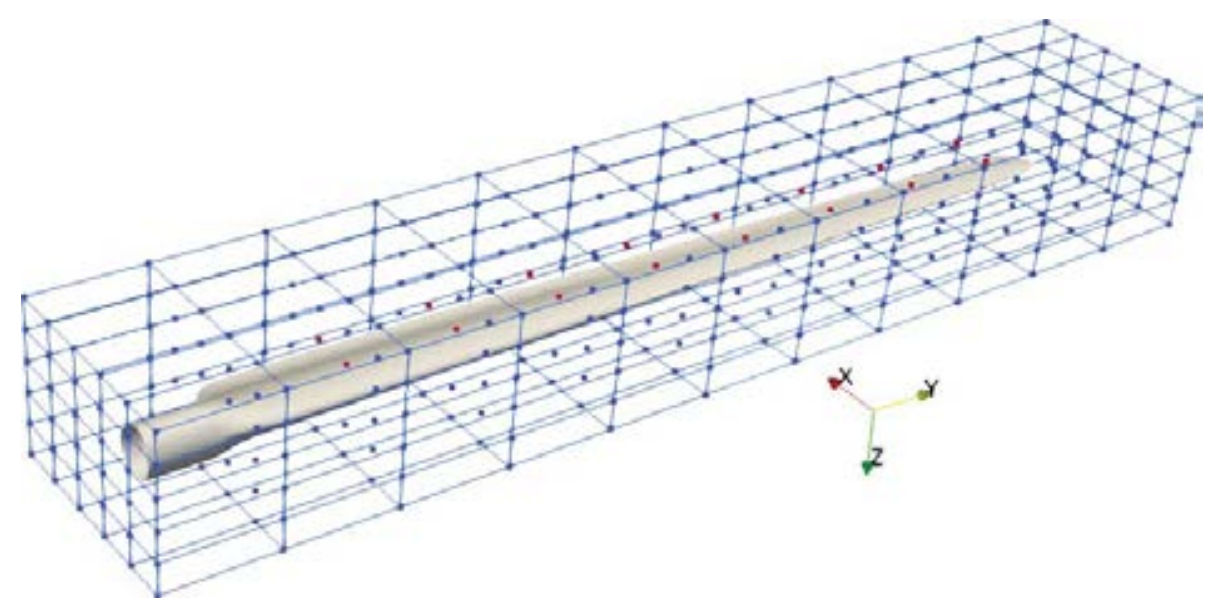

Figure 4: MEXICO WT Blade: A $6 \times 12 \times 6$ volumetric B-Splines morphing box parameterizes the blade and part of the mesh. Control points (CPs) in red were allowed to move only in the axial direction $(\mathrm{z})$; CPs in blue remain frozen during the optimization.

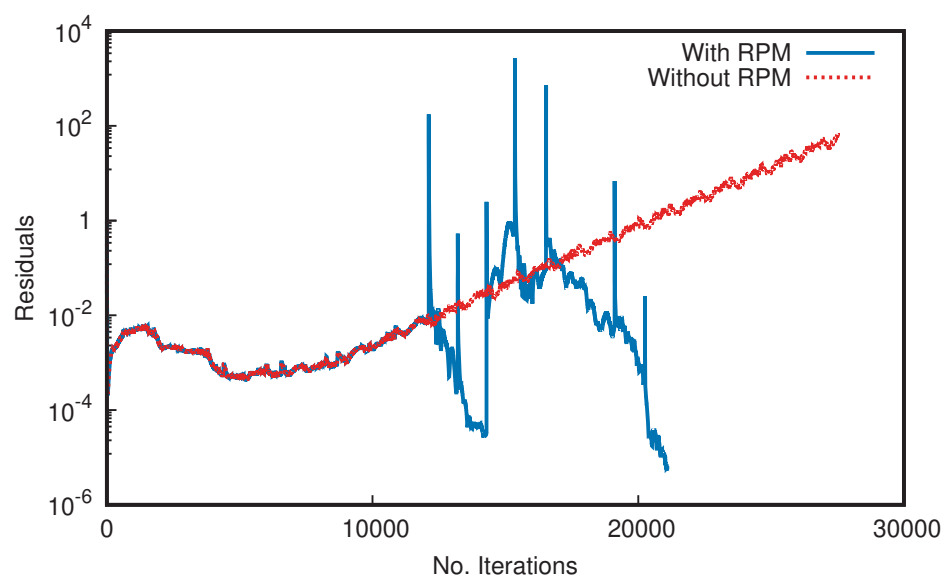

Figure 5: MEXICO WT Blade: Convergence of the adjoint equations with/without the RPM (blue/red) within the first optimization cycle. Residuals averaged over the ensemble of equations are plotted in logarithmic scale.

From Figs. 6, it can be observed that the optimizer has mainly changed the shape of the blade close to its tip. Smaller changes can also be seen close to the root. Fig. 7 demonstrates that the optimizer bended the tip towards the axial flow direction and slightly increased the blade yaw angle at the same position. 


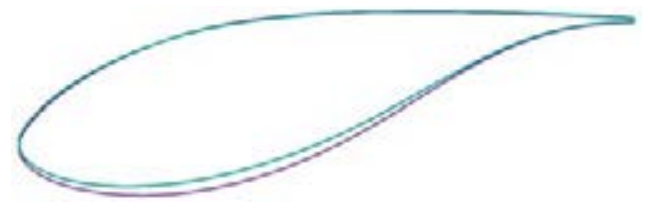

(a) $25 \%$ Span

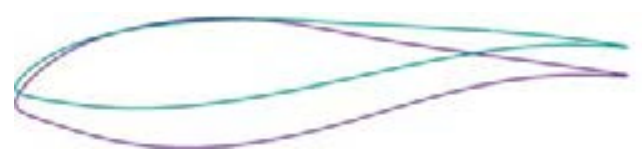

(c) $60 \%$ Span

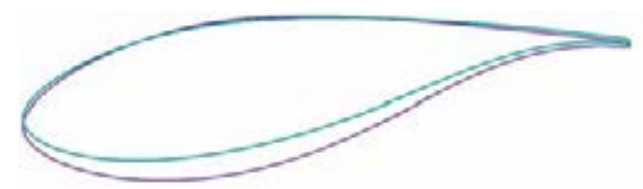

(b) $35 \%$ Span

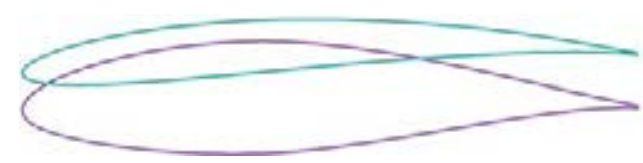

(d) $82 \%$ Span

Figure 6: Comparison of the baseline (magenta) and optimized (green) blade sections at a number of spanwise positions.

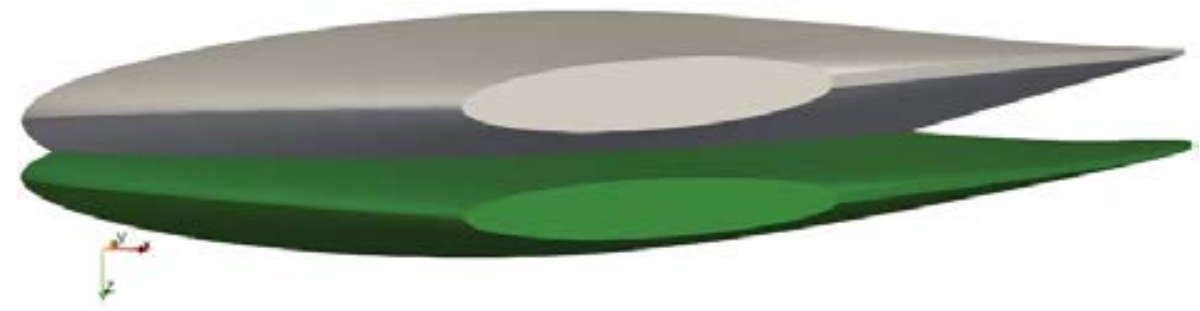

Figure 7: Comparison of the baseline (white) and optimized (green) geometries of the blade, as seen from its tip.

To get a clearer view on local deformations along the blade span, the cumulative normal displacement of the optimized blade surface is presented in Figs. 8.

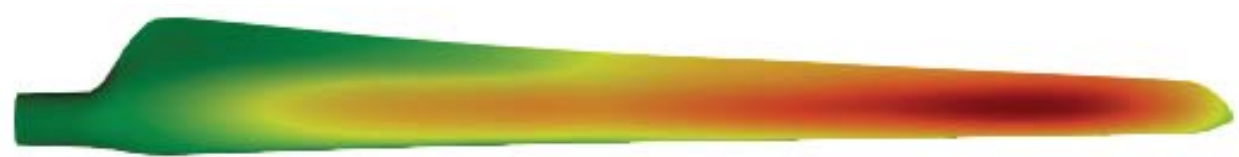

(a) Pressure side

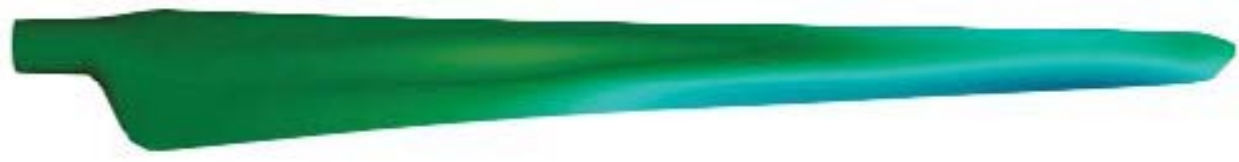

(b) Suction side

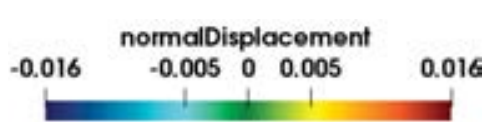

Figure 8: Cumulative normal displacements of the blade surface. Positive/negative signs (red/blue) indicate inward/outward displacements. 
The comparison of the pressure coefficient distribution on the blade for the baseline and optimized geometries is presented in Figs. 9 and 10 for the pressure and suction sides, respectively. Figs. 10a and 10b demonstrate that the largest pressure coefficient difference is at the middle and upper part of the blade, whereas the pressure coefficient at the leading and trailing edges does not change significantly w.r.t. the baseline design. For the pressure side of the blade, Fig. 9a and $9 \mathrm{~b}$ show that the significant pressure coefficient difference happens at the upper part of the blade.

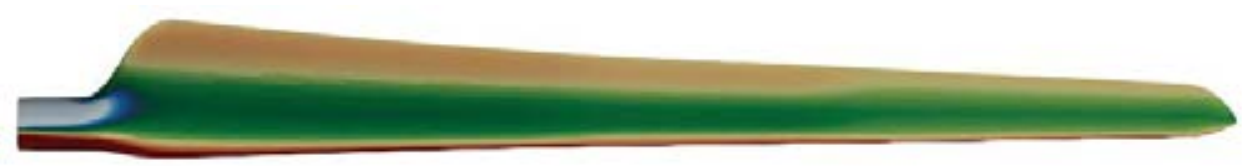

(a) Baseline

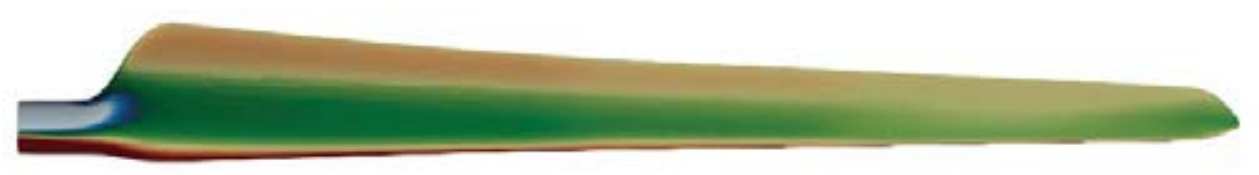

(b) Optimized

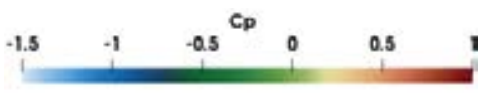

Figure 9: Comparison of the pressure coefficient distribution on the blade pressure side.

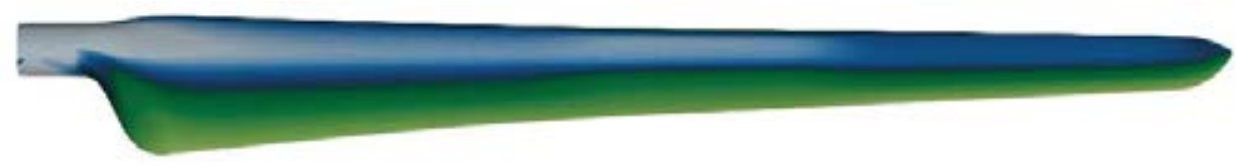

(a) Baseline

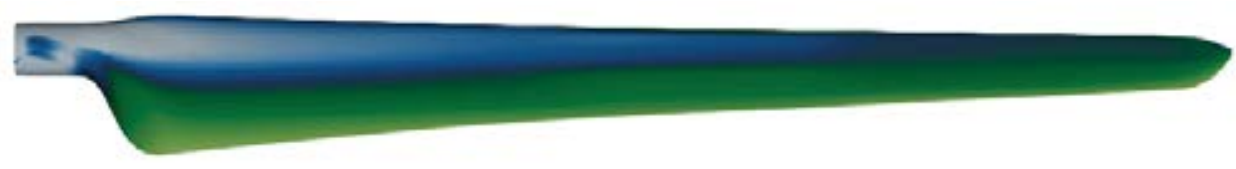

(b) Optimized

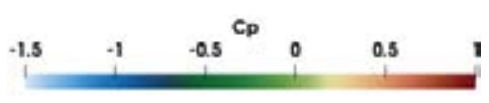

Figure 10: Comparison of the pressure coefficient distribution on the blade suction side.

The pressure coefficient distributions of the baseline and optimized geometries are also plotted in Fig. 11 at a number of spanwise positions. It is evident that the largest increase in the objective function comes from the tip, whereas the pressure coefficient is almost unchanged close to its root. 


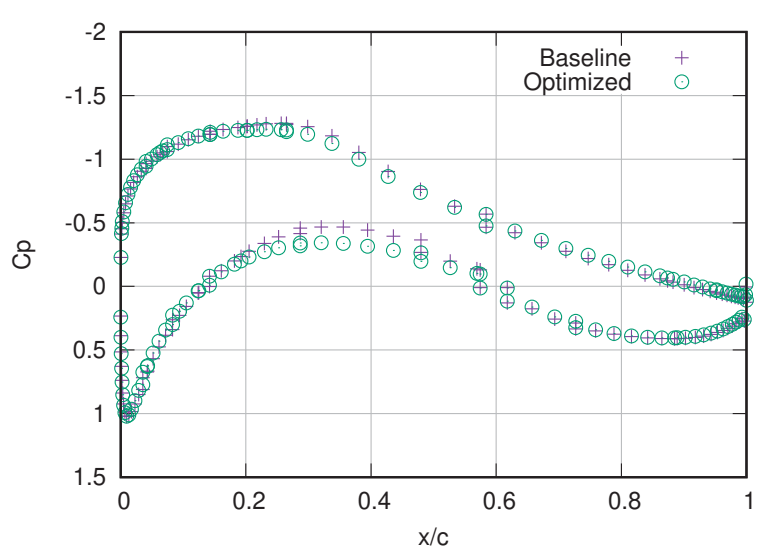

(a) $25 \% \mathrm{Span}$

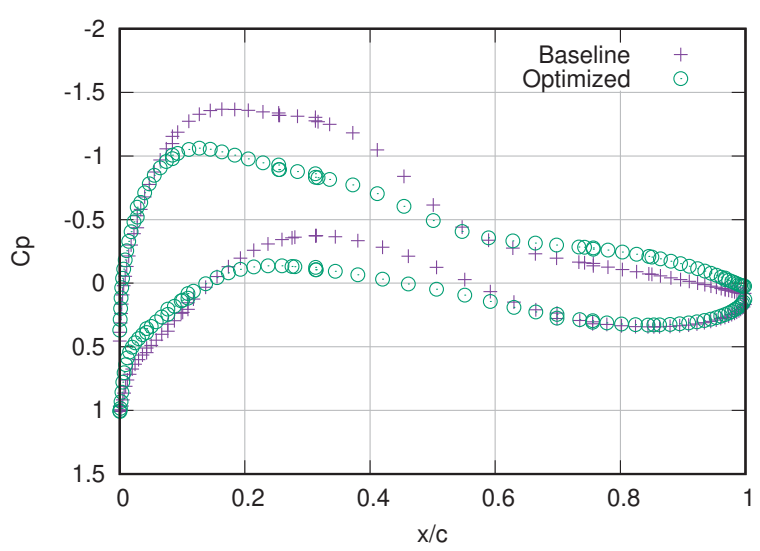

(c) $60 \%$ Span

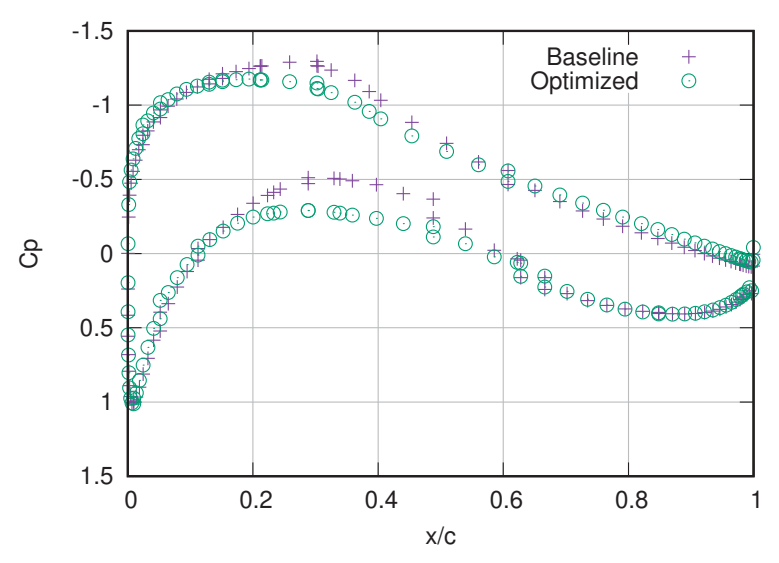

(b) $35 \%$ Span

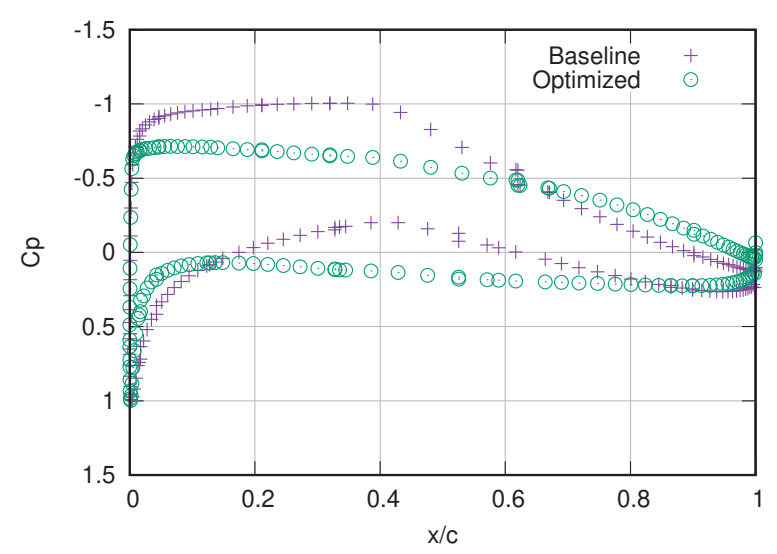

(d) $82 \%$ Span

Figure 11: Comparison of the pressure coefficient of the baseline and optimized blades at four spanwise positions.

\section{Conclusions}

This paper presented an application of the publicly available adjointOptimisationFoam, the adjoint-based optimizer within OpenFOAM developed by the authors, to the optimization of the MEXICO wind turbine, targeting an increased axial moment coefficient. The capabilities of the software were enhanced by including the adjoint to the terms taking the rotation of the computational domain into consideration. Convergence difficulties, encountered in the numerical solution of the adjoint equations, were treated using the RPM which enabled the adjoint solver to converge and compute the required sensitivities. The application of the extended software led to an increase in the axial moment coefficient by $10.8 \%$, by mainly bending the blade tip in the axial direction and changing the blade yaw angle close to the tip.

\section{Acknowledgments}

This project has received funding from the European Union's Horizon 2020 research and innovation program under the Marie Sklodowska Curie Grant Agreement No 860101 (zEPHYR). 


\section{REFERENCES}

[1] T. Liu, P.J. Tavner, Y. Feng, and Y.N. Qiu. Review of recent offshore wind power developments in China. Wind Energy, 16(5):786-803, 2013.

[2] T. Ashuri and MB. Zaayer. Size effect on wind turbine blade's design drivers. In Proceedings of the European Wind Energy Conference and Exhibition EWEC, Brussels, 2008.

[3] J.R.R.A. Martins and A.B. Lambe. Multidisciplinary design optimization: A survey of architectures. AIAA Journal, 51(9):2049-2075, 2013.

[4] H. Glauert. The elements of aerofoil and airscrew theory. Cambridge University Press, 1983.

[5] S. Chen, Z. Lyu, G.K.W. Kenway, and J.R.R.A. Martins. Aerodynamic shape optimization of common research model wing-body-tail configuration. Journal of Aircraft, 53(1):276$293,2016$.

[6] E.M. Papoutsis-Kiachagias, V.G. Asouti, K.C. Giannakoglou, K. Gkagkas, S. Shimokawa, and E. Itakura. Multi-point aerodynamic shape optimization of cars based on continuous adjoint. Structural and Multidisciplinary Optimization, 59(2):675-694, 2019.

[7] M.A. Elfarra, N. Sezer-Uzol, and I.S. Akmandor. NREL VI rotor blade: Numerical investigation and winglet design and optimization using CFD. Wind Energy, 17(4):605-626, 2014.

[8] J.E.V. Peter and R.P. Dwight. Numerical sensitivity analysis for aerodynamic optimization: A survey of approaches. Computers \& Fluids, 39(3):373-391, 2010.

[9] E.M. Papoutsis-Kiachagias and K.C. Giannakoglou. Continuous adjoint methods for turbulent flows, applied to shape and topology optimization: industrial applications. Archives of Computational Methods in Engineering, 23(2):255-299, 2016.

[10] R. Ritlop and S. Nadarajah. Design of wind turbine profiles via a preconditioned adjointbased aerodynamic shape optimization. 47th AIAA Aerospace Sciences Meeting including The New Horizons Forum and Aerospace Exposition, pages 2009-1547, 2009.

[11] T. Dhert, T. Ashuri, and J.R.R.A. Martins. Aerodynamic shape optimization of wind turbine blades using a reynolds-averaged Navier-Stokes model and an adjoint method. Wind Energy, 20(5):909-926, 2017.

[12] K.T. Tsiakas, X.S. Trompoukis, V. Asouti, and K.C. Giannakoglou. Shape optimization of wind turbine blades using the continuous adjoint method and volumetric NURBS on a GPU cluster. In Advances in Evolutionary and Deterministic Methods for Design, Optimization and Control in Engineering and Sciences, pages 131-144. Springer, 2019.

[13] G.M. Shroff and H. Keller. Stabilization of unstable procedures: The Recursive Projection Method. SIAM Journal of Numerical Analysis, 30(4):1099-1120, 1993.

[14] P. Spalart and S. Allmaras. A one-equation turbulence model for aerodynamic flows. AIAA Paper No. 92-0439, 1992. 
[15] P. Tucker. Differential equation-based wall distance computation for DES and RANS. Journal of Computational Physics, 190:229-248, 2003.

[16] D. Papadimitriou and K. Giannakoglou. A continuous adjoint method with objective function derivatives based on boundary integrals for inviscid and viscous flow. Computers \& Fluids, 36(2):325-341, 2007.

[17] A.S. Zymaris, D.I. Papadimitriou, K.C. Giannakoglou, and C. Othmer. Continuous adjoint approach to the Spalart-Allmaras turbulence model for incompressible flows. Computers \& Fluids, 38(8):1528-1538, 2009.

[18] S. Patankar. Numerical Heat Transfer and Fluid Flow. Hemisphere, Taylor \& Francis Group, 1st edition, 1980.

[19] R. Dwight and D. Vollmer. Efficient algorithms for solution of the adjoint compressible Navier-Stokes equations with applications. In Proceedings of the ONERA-DLR Aerospace Symposium (ODAS), Toulouse, 2006.

[20] J.G. Schepers and H. Snel. Model experiments in controlled conditions. Technical Report E-07-042, Energy Research Center of the Netherlands, 2007.

[21] H. Snel, J.G. Schepers, and L. Pascal. First results from mexnext: Analysis of detailed aerodynamic measurements on a 4.5 diameter rotor placed in the large German Dutch Wind Tunnel DNW. In European Wind Energy Conference and Exhibition (EWEC), Warsaw, Poland, 2010.

[22] G. Papadakis, S. Voutsinas, G. Sieros, and T. Chaviaropoulos. CFD aerodynamic analysis of non-conventional airfoil sections for very large rotor blades. Journal of Physics: Conference Series, 555 012104, 2014.

[23] J.G. Schepers, K. Boorsma, T. Cho, S. Gomez-Iradi, P. Schaffarczyk, WZ. Shen, T. Lutz, B. Stoevesandt, S. Schreck, D. Micallef, et al. Analysis of MEXICO wind tunnel measurements. Final report of IEA task 29, Mexnext (phase 1). Technical Report E-12-004, ECN, 2012. 